\title{
Role of (1-3)-B-D-Glucan Test in the Diagnosis of Invasive Fungal Infections among High-Risk Patients in a Tertiary Care Hospital
}

\author{
Tanureet Kaur Sandhar ${ }^{1}$ Deepinder Kaur Chhina ${ }^{1}$ Veenu Gupta ${ }^{1}$ Jyoti Chaudhary ${ }^{1}$ \\ ${ }^{1}$ Department of Microbiology, Dayanand Medical College and \\ Hospital, Ludhiana, Punjab, India \\ Address for correspondence Jyoti Chaudhary, MBBS, MD, 1201, \\ Mulberry-3, Centra Greens Apartment, Pakhowal Road, Ludhiana, \\ Punjab, 141013, India (e-mail: drjyotisohu@gmail.com). \\ J Lab Physicians 2022;14:306-311.
}

\begin{abstract}
Background Invasive fungal infections (IFI) are associated with high mortality. Serum fungal biomarkers offer an advantage over the traditional methods in early diagnosis and better clinical outcomes. The aim of the study was to evaluate the role of (1-3)- $\beta$-Dglucan (BDG) assay in the patients suspected of IFI.

Materials and Methods This prospective study was conducted in the Department of Microbiology, Dayanand Medical College and Hospital, Ludhiana, over a period of 1 year. A total of 862 serum samples were received from patients suspected of IFI, for the BDG test (Fungitell, Associates of Cape Cod Inc., USA). The test was performed as per kit protocol. Appropriate samples were processed for $\mathrm{KOH}$ fungal smear and fungal culture. Blood culture was done by Bactec (Biomerieux).

Statistical Analysis Results were analyzed using descriptive statistical methods. Sensitivity, specificity, accuracy, positive predictive value (PPV), and negative predictive value (NPV) were calculated at different cutoffs. In addition, the receiver operating characteristic (ROC) curve using SPSS 21.00 software was calculated and the diagnostic accuracy was shown by the area under the ROC curve (AUC).

Results Among 862 patients, 546 (63.3\%) were males. The predominant age group (25.6\%) was between 61 and 70 years. The most common risk factor $(54.8 \%)$ was prolonged intensive care unit stay. Out of the total samples, $455(52.8 \%)$ samples were found positive for BDG. Fungal elements were seen in $48(10.5 \%) \mathrm{KOH}$ smears and fungal growth was obtained in $81(17.8 \%)$ cultures. Comparison of BDG assay and culture at different cutoffs yielded AUC -0.823 . Sensitivity (100\%), specificity (51.3\%), accuracy (55.6\%), PPV (15.8\%), and NPV (100\%) were observed at the kit cutoff of $80 \mathrm{pg} / \mathrm{mL}$. Optimum sensitivity and specificity of $79.2 \%$ and $70.3 \%$, respectively, were observed at a cutoff of $142.4 \mathrm{pg} / \mathrm{mL}$. A

Keywords

- invasive fungal infections

- (1-3)- $\beta$-D-glucan

- fungal culture significant correlation was observed between BDG positivity and piperacillin-tazobactam use and dialysis. Among BDG positive patients, 38(8.4\%) succumbed to death.

Conclusion Detection of BDG helps in the early diagnosis of IFI in critically ill patients. As the assay has a high NPV, a negative test can be used to stop the empirical antifungal drugs. The use of a higher cutoff can be useful to avoid false-positive results.
\end{abstract}

published online February 9, 2022
DOI https://doi.org/ $10.1055 / \mathrm{s}-0042-1742632$. ISSN $0974-2727$. (c) 2022. The Indian Association of Laboratory Physicians. All rights reserved.

This is an open access article published by Thieme under the terms of the Creative Commons Attribution-NonDerivative-NonCommercial-License, permitting copying and reproduction so long as the original work is given appropriate credit. Contents may not be used for commercial purposes, or adapted, remixed, transformed or built upon. (https://creativecommons.org/ licenses/by-nc-nd/4.0/)

Thieme Medical and Scientific Publishers Pvt. Ltd., A-12, 2nd Floor, Sector 2, Noida-201301 UP, India 


\section{Introduction}

Invasive fungal infections (IFI) are increasing worldwide and are associated with high mortality, particularly in the immunocompromised population. Various risk factors are associated with IFI, including neutropenia, prolonged hospital stay, human immunodeficiency virus (HIV) infection, hematological malignancies, bone marrow transplantation, solid organ transplantation, chemotherapy, and the use of immunosuppressive agents. ${ }^{1}$ The predominant fungi responsible for IFI are Candida species (spp.) and Aspergillus spp., followed by Crypococcus spp., Mucormycetes spp., Fusarium spp., Scedosporium spp., etc. ${ }^{2}$ Invasive candidiasis (40-50\%) and invasive Aspergillosis (30-70\%) in intensive care units (ICUs) is associated with high mortality if not treated timely. ${ }^{3}$ Diagnosis of IFI is still a challenge. Currently, it is based on radiological examination, histopathological examination and fungal culture. Radiological findings are nonspecific; fungal culture usually takes 2 to 7 days to obtain results, while a histological examination is difficult to achieve in most cases as it is an invasive procedure. ${ }^{4}$ Therefore, a simple and convenient method is needed for rapid diagnosis and better management of these deadly IFI.

Presence of various fungal antigens or metabolites in blood correlates with IFI. Various nonculture techniques have been described to detect these biomarkers. However, these tests are routinely not performed in most clinical laboratories as they require specialized equipment and the cost of the tests is high. ${ }^{5}$ The tests detecting (1-3)- $\beta$-D-glucan (BDG) and galactomannan (GM) have been included in the revised criteria for diagnosis of IFI by the European Organization for Research and Treatment of Cancer/Mycoses Study Group. ${ }^{6}$

BDG is a component of the cell wall of many pathogenic fungi except Cryptococcus neoformans and Mucormycetes. Its presence in the bloodstream correlates with IFI caused by Candida spp., Aspergillus species, Pneumocystis jirovecii, dimorphic fungus such as Histoplasma capsulatum, Coccidioides immitis, Sporothrix schenckii, Fusarium spp., Trichosporon species, Acremonium species, and Saccharomyces cerevisiae. The yeast phase of Blastomyces dermatitidis produces very little BDG, which is not detectable by the assay. The kinetic assay is based upon a modification of the limulus amebocyte lysate pathway detects BDG by colorimetric or turbidometric method., 2,7,8 Currently, two kits, including the Fungitell Assay (Associates of Cape Cod, Inc, Falmouth, Massachusetts, United States) and Wako $\beta$ glucan test (Fujifilm Wako Pure Chemical crop. Tokyo, Japan), are commercially available for BDG testing. ${ }^{2}$

High sensitivity and specificity of BDG assay reported by various studies and its prognostic use have also been evaluated by a few. ${ }^{2}$ Though some confounding factors responsible for false positivity have been observed including broadspectrum antibiotics, dialysis and use of immunoglobulins. ${ }^{9,10}$ Most of the studies done on the role of BDG assay for the diagnosis of IFI are from western countries. Very few studies have been reported from India. ${ }^{6}$ Therefore, this study was planned to evaluate BDG assay in adult patients with high risk of IFI.

\section{Materials and Methods}

It was a prospective study conducted over a period of 1 year from January 2019 to December 2019 in the Department of Microbiology, Dayanand Medical College and Hospital, Ludhiana. The study was approved by the ethical committee of the hospital. Admitted patients over 18 years of age, suspected of IFI whose requisitions for BDG test were received in the Department of Microbiology, were included in the study. Demographic and clinical details of the patients were recorded. Appropriate clinical samples were processed and subjected to microscopy ( $\mathrm{KOH}$ wet mount), fungal culture on Sabouraud dextrose agar (SDA), automated blood culture (BacT/ALERT, Biomerieux), and BDG assay.

\section{Fungal Culture}

The samples such as sputum, endotracheal secretions (ET secretions) and various fluids such as bronchoalveolar lavage fluid (BAL), ascitic fluid, pleural fluid, drain fluid, bile fluid, and biopsy tissue were inoculated on two sets (with cycloheximide and without cycloheximide) of the SDA tubes. One set of tubes was incubated at $25^{\circ} \mathrm{C}$ and the other set was incubated at $37^{\circ} \mathrm{C}$. The inoculated media were kept for a minimum period of 3 weeks. Tubes were examined daily during first week and on alternate days in the second and third week. The growth obtained was identified on the basis of colony morphology and microscopic examination of lactophenol cotton blue preparation, germ tube test, and biochemical reactions.

\section{Blood Culture}

Five to ten milliliters of blood were inoculated into blood culture bottles. The bottles were incubated in an automated system (BacT/ALERT, Biomerieux) system and monitored for positivity. Bottles that did not show growth for 5 days were discarded. The positive bottles were subcultured on blood agar and McConkey agar, and the yeast-like growth obtained after 24 to 48 hours of incubation was further identified by the vitek2 system.

\section{(1-3)- $\beta$-D Glucan (BDG) Assay (Fungitell)}

Serum was separated and BDG test was done using U.S. Food and Drug Administration approved Fungitell kit (Associates of Cape Cod). The test procedure was done as per the kit protocol. The Fungitell test results were interpreted as negative-BDG values of less than $60 \mathrm{pg} / \mathrm{mL}$, indeterminate valued of 60 to $79 \mathrm{pg} / \mathrm{mL}$, and positive values of greater than or equal to $80 \mathrm{pg} / \mathrm{mL}$.

Sensitivity specificity, positive predictive value (PPV), negative predictive values (NPV), and area under the receiver operating characteristic (ROC) curve were calculated by comparing BDG assay with culture.

\section{Results}

A total of 899 blood samples from 862 patients admitted with suspicion for IFI were received for BDG testing and two BDG test requisitions were received from 37 patients. Among these patients, males (546; 63.3\%) were more as compared 
Table 1 Distribution of various risk factors among the patients suspected for IFI $(n=862)$

\begin{tabular}{|l|l|l|l|}
\hline Risk factors & $\begin{array}{l}\text { BDG positive } \\
(\boldsymbol{n}=\mathbf{4 5 5})\end{array}$ & $\begin{array}{l}\text { BDG negative } \\
(\boldsymbol{n}=\mathbf{4 0 7})\end{array}$ & $p$-Value \\
\hline Steroid use & $159(34.9 \%$ & $151(37.1 \%)$ & 0.805 \\
\hline Malignancy & $16(3.5 \%)$ & $17(4.2 \%)$ & 0.876 \\
\hline HIV & $3(0.65 \%)$ & $1(0.25 \%)$ & 0.622 \\
\hline $\begin{array}{l}\text { Organ } \\
\text { transplant }\end{array}$ & $6(1.32 \%)$ & $6(1.5 \%)$ & 0.920 \\
\hline Neutropenia & $96(21.1 \%)$ & $57(14 \%)$ & 0.064 \\
\hline $\begin{array}{l}\text { ICU stay } \\
>7 \text { days }\end{array}$ & $243(53.4 \%)$ & $229(56.2 \%)$ & 0.226 \\
\hline
\end{tabular}

Abbreviations: BDG, (1-3)- $\beta$-D-glucan; ICU, intensive care unit; IFI, invasive fungal infections.

with females ( $316 ; 6.7 \%)$. The predominant age group (25.6\%) was 61 to 70 years. Out of the total cases, 613 (71.1\%) were admitted in ICUs, and 249 (28.9\%) were in wards. Among all enrolled patients, 420 (49\%) had immunocompromised status. The most common risk factor for IFI was prolonged ( $>7$ days) ICU stay $(472 ; 54.8 \%$ ) followed by steroid use (310; 36\%), neutropenia (153; 17.7\%), malignancy (33; 3.8\%), organ transplant $(12 ; 1.4 \%)$, and HIV $(4 ; 0.5 \%)$ (- Table 1 ).

Out of 862 patients tested for BDG levels, blood for culture was received from 853 patients. Other samples received for fungal culture were sputum (439), ET secretions (346), BAL (1), ascitic fluid (33), pleural fluid (16), drain fluid (5), bile fluid (2), and biopsy (12).

Out of 862 samples received for BDG testing, 455 (52.8\%) samples were found positive, $92(10.7 \%)$ were indeterminate, and 315 (36.5\%) were found negative. The first positive sample was included if repeat test received from the same patient. Among positive samples, 273 (60\%) were from male patients, and most of these were admitted to ICU (71.9\%) (-Fig. 1). Fungal elements were seen in 48 (10.5\%) $\mathrm{KOH}$ smears, and fungal growth was obtained from 81 (17.8\%)

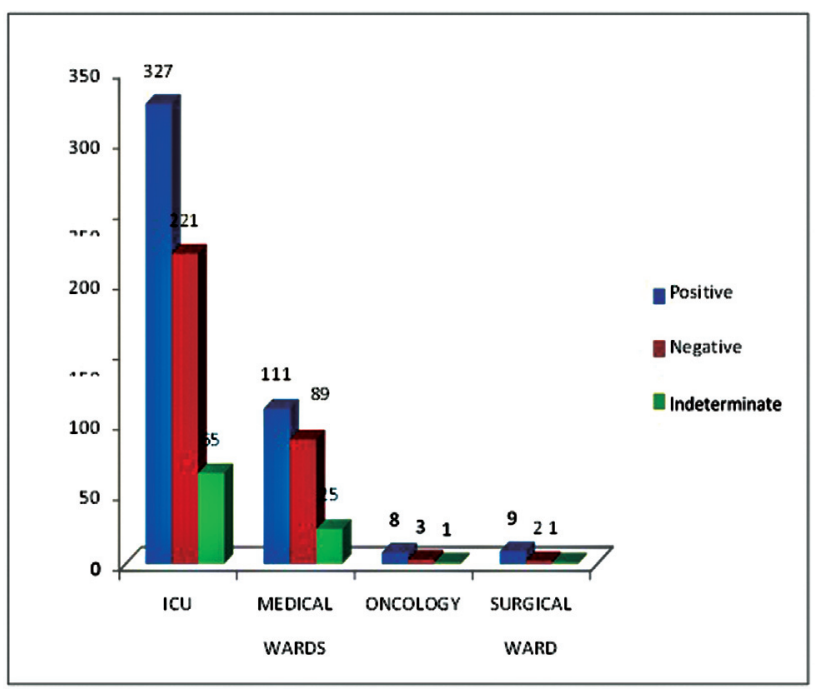

Fig. 1 Area wise distribution of (1-3)- $\beta$-D-glucan assay positivity $(n=862)$. ICU, intensive care unit.
Table 2 Comparative evaluation of (1-3)-B-D-glucan, blood culture, and fungal culture $(n=455)$

\begin{tabular}{|l|l|l|l|}
\hline $\begin{array}{l}\text { Cutoff } \\
(\mathrm{pg} / \mathrm{mL})\end{array}$ & $\begin{array}{l}(1-3)-\beta-D-g l u c a n \\
\text { assay (\%) }\end{array}$ & $\begin{array}{l}\text { Blood } \\
\text { culture (\%) }\end{array}$ & $\begin{array}{l}\text { Fungal } \\
\text { culture (\%) }\end{array}$ \\
\hline $80-100$ & $69(15)$ & $3(4.3)$ & $2(2.9)$ \\
\hline $100-200$ & $179(39.3)$ & $9(5.0)$ & $15(8.4)$ \\
\hline $200-300$ & $68(14.9)$ & $8(11.7)$ & $7(10.2)$ \\
\hline $300-400$ & $35(7.7)$ & $3(8.5)$ & $5(14.2)$ \\
\hline $400-500$ & $21(4.6)$ & $3(14.2)$ & $3(14.2)$ \\
\hline$>500$ & $83(18.2)$ & $12(14.5)$ & $11(13.2)$ \\
\hline Total & $455(100)$ & $38(8.4)$ & $43(9.5)$ \\
\hline
\end{tabular}

samples, including 38 (8.4\%) blood cultures and 43 (9.5\%) other samples (-Table 2). Candida spp. isolated from the sputum and ET samples were considered as colonizers. A total of 56 (69\%) Candida spp. and 25 (31\%) Aspergillus spp. (20 Aspergillus flavus and 5 Aspergillus fumigatus) were isolated (-Table 3). Among Candida spp., 9 (16\%) were Candida albicans, and 47(84\%) nonalbicans. Candida spp. included C. tropicalis (35; 62.5\%), C. parapsilosis (7; 12.5\%), C. stellatoidea ( $3 ; 5.4 \%)$, C. lusitaniae $(1 ; 1.8 \%)$, and C. glabrata (1; $1.8 \%)$. Among BDG negative and indeterminate patients, no significant fungal growth was obtained in culture. Though the growth of Candida spp. obtained from 30 sputum samples (21 BDG negative +9 BDG indeterminate) and 12 ET aspirates (6 BDG negative +6 BDG indeterminate) were considered as colonizers.

The ROC curve using SPSS 21.00 software was calculated by plotting true positive results (sensitivity) versus false positive (1-specificity) at various cutoff points. The diagnostic accuracy given by the area under the ROC curve (AUC) was 0.823 . The ROC revealed the BDG assay sensitivity (100\%), specificity (51.3\%), accuracy (55.6\%), PPV (15.8\%), and NPV (100\%) at the kit cutoff of greater than or equal to $80 \mathrm{pg} / \mathrm{mL}$. The specificity increases as we increase the cutoff

Table 3 Distribution of fungal isolates in BDG-positive patients $(n=455)$

\begin{tabular}{|l|l|l|}
\hline Samples & $\begin{array}{l}\text { Candida } \\
\text { species }\end{array}$ & $\begin{array}{l}\text { Aspergillus } \\
\text { species }\end{array}$ \\
\hline Sputum $(n=220)$ & $76(34.5 \%)^{\text {a }}$ & $11(5 \%)$ \\
\hline $\begin{array}{l}\text { Endotracheal } \\
\text { secretions }(n=206)\end{array}$ & $72(34.9 \%)^{\text {a }}$ & $14(6.8 \%)$ \\
\hline Ascitic fluid $(n=12)$ & $5(41.7 \%)$ & - \\
\hline Drain fluid $(n=2)$ & $2(100 \%)$ & - \\
\hline Biopsy $(n=9)$ & $9(100 \%)$ & - \\
\hline Bile fluid $(n=1)$ & - & - \\
\hline Pleural fluid $(n=5)$ & $2(40 \%)$ & - \\
\hline Total $(n=455)$ & 166 & 25 \\
\hline
\end{tabular}

Abbreviation: BDG, (1-3)- $\beta$-D-glucan. ${ }^{\mathrm{a} C o l o n i z e r s . ~}$ 
Table 4 Sensitivity, specificity, and predictive values of (1-3)- $\beta$-D-glucan assay at different cutoff points $(n=455)$

\begin{tabular}{|l|l|l|l|l|l|}
\hline $\begin{array}{l}(1-3)-\beta-D-g l u c a n \\
\text { assay } \\
\text { cutoffs }\end{array}$ & Sensitivity & Specificity & $\begin{array}{l}\text { Positive predictive } \\
\text { value }\end{array}$ & $\begin{array}{l}\text { Negative predictive } \\
\text { value }\end{array}$ & Accuracy \\
\hline$>80$ & $100.00 \%$ & $51.52 \%$ & $15.82 \%$ & $100.00 \%$ & $55.57 \%$ \\
\hline$>100$ & $93.06 \%$ & $59.62 \%$ & $17.36 \%$ & $98.95 \%$ & $62.41 \%$ \\
\hline$>200$ & $62.50 \%$ & $79.49 \%$ & $21.74 \%$ & $95.88 \%$ & $78.07 \%$ \\
\hline$>300$ & $47.22 \%$ & $86.71 \%$ & $24.46 \%$ & $94.74 \%$ & $83.41 \%$ \\
\hline$>400$ & $38.89 \%$ & $90.38 \%$ & $26.92 \%$ & $94.20 \%$ & $86.08 \%$ \\
\hline$>500$ & $30.56 \%$ & $92.28 \%$ & $26.51 \%$ & $93.58 \%$ & $87.12 \%$ \\
\hline
\end{tabular}

Abbreviation: BDG, (1-3)- $\beta$-D-glucan.

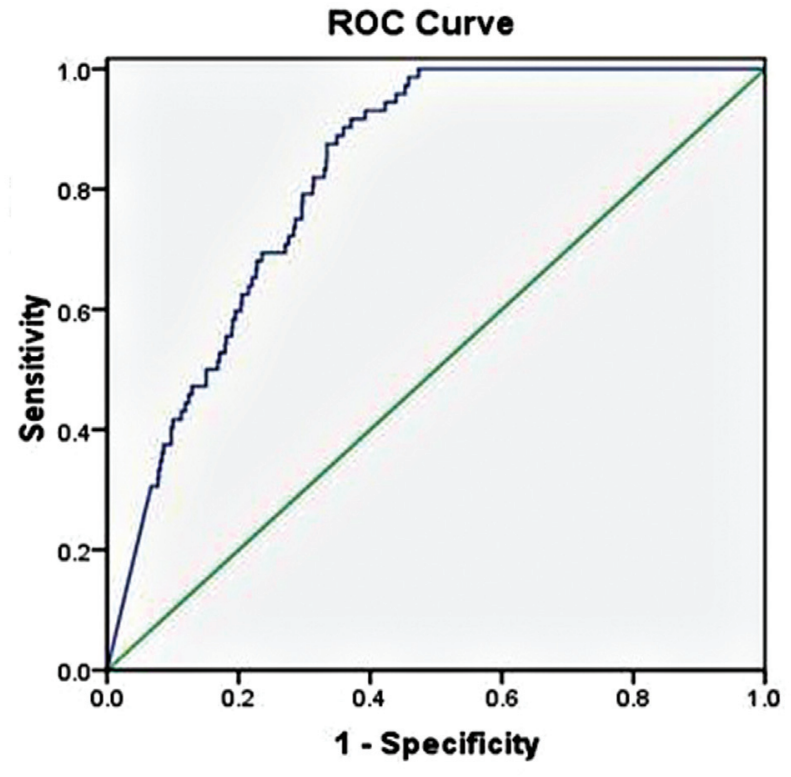

Diagonal segments are produced by ties.

Fig. 2 Receiver operating characteristic (ROC) curve.

that parallels with decrease in sensitivity ( - Table 4). Optimum sensitivity and specificity of $79.2 \%$ and $70.3 \%$, respectively, were observed at a cutoff of $142.4 \mathrm{pg} / \mathrm{mL}$ ( - Fig. 2).

Among 455 positive patients, 205 (45.1\%) were on piperacillin-tazobactam (PTZ). A significant correlation was observed between BDG positivity and PT use ( $p$-value $<0.05$ ). Patients on dialysis (17.1\%) also showed a significant correlation with the BDG positivity. No significant correlation was observed with the use of amoxicillin-clavulanic acid 178 (39.1\%).

A repeat sample for the BDG test was received from 37 positive patients. Out of these, 24 patients were found positive, 10 patients were found negative, and 3 showed indeterminate results on a repeat test. Among 37 patients, 17 patients were on antifungal treatment. Out of these, 15 patients showed a decrease in BDG levels and 2 patients did not show response to therapy. Out of the 15 patients who has shown decreased BDG levels, 13 patients were dis- charged. Out of 455 positive patients, 359 (78.9\%) patients were discharged, and 38 (8.4\%) succumbed to death.

\section{Discussion}

Currently, particular emphasis is placed on the detection of fungal biomarkers to improve the diagnosis of IFI. In the present study of 862 consecutive cases of suspected IFI, the mean age of the patients was $56.16 \pm 16.71$ (range: 19-101 years). Similar observations were made by other studies. ${ }^{11}$ However, Singh et al reported median age of 33 years. ${ }^{6}$ We have observed the male predominance (63.3\%) among suspected IFI patients that is in consistent with other studies. ${ }^{6,12}$ This may be explained by the fact that males are maximally exposed to fungal spores, particularly working with decaying vegetation like moldy hay in agriculture.

Studies have shown that a longer median duration ( $>7$ days) of stay in ICU is associated with IFI. ${ }^{12}$ A significant association was not observed with prolonged ICU stay in our study, though it was the most common risk factor as our institute is a tertiary care center in Punjab and critically ill patients from all other hospitals are referred here. A study from PGI Chandigarh reported prolonged antibiotic intake (60\%) as the most common risk factor for IFI followed by ICU stay (42.8\%). ${ }^{6}$ However, Herbrecht et al have reported hematological malignancies (63.2\%) as the most common risk factor. ${ }^{13}$

BDG assay helps to detect IFI at an early stage before being detected by other methods and guide for the empirical antifungal treatment. There are very few studies available on BDG assay from India. The reason might be the high cost of the test. In the present study, BDG assay positivity was observed as $52.8 \%$. The results were consistent with the study by Singh et al, and they have reported $57.1 \%$ positivity of BDG assay among the total samples. ${ }^{6}$ However, Bansal et al have reported $90 \%$ positivity among confirmed candidemia cases from ICU settings. ${ }^{14}$

Though fungal culture is a gold standard test, it reported a low positivity rate of $50 \%$ with best culture techniques in suspected IFI cases..$^{15}$ In the present study, the fungal smear was positive in 48 (10.5\%) of the samples, while fungal culture positivity was observed in 81 (17.8\%) of the BDG 
positive samples. A significant correlation was observed between BDG positive samples and fungal culture positivity with $p$-values less than 0.05 . In the study conducted by Cordonnier et al, septate hyphae were seen in $6.6 \%$ fungal smears and growth was obtained in $71.4 \%$ of the fungal cultures. ${ }^{16}$ Another study on invasive aspergillosis showed $21.9 \%$ fungal smear positivity, and $19.4 \%$ samples showed fungal growth in culture. ${ }^{17}$ Similarly, a study on invasive candidiasis reported $19 \%$ blood culture positivity. ${ }^{18}$

Studies on IFI have reported BDG positive results in almost all the candidemia patients. ${ }^{19}$ In our study, the ROC curve was analyzed at various cutoff points. The diagnostic accuracy given by the AUC was 0.823 , which showed sensitivity of $100 \%$ and specificity of $51.5 \%$, PPV of $15.82 \%$, NPV of $100 \%$, and accuracy of $55.6 \%$ at kit cutoff of greater than $80 \mathrm{pg} / \mathrm{mL}$. When cutoff was increased from $80 \mathrm{pg} / \mathrm{mL}$, specificity improved. Optimum sensitivity and specificity of 79.2 and $70.3 \%$, respectively, were observed at a cutoff of 142.4 $\mathrm{pg} / \mathrm{mL}$. Congruous with our findings, Bansal et al reported the sensitivity and specificity of the BDG assay $97.8 \%$ and $16.6 \%$, respectively, at cutoff of $80 \mathrm{pg} / \mathrm{mL}$ and 97.8 and $63.3 \%$ at a cutoff of $143.5 \mathrm{pg} / \mathrm{mL}($ AUC $=0.908) .{ }^{14}$ However, a study has shown higher diagnostic accuracy of 0.995 with a sensitivity of $95.1 \%$ and specificity of $96 \%$ at the kit cutoff of $80 \mathrm{pg} / \mathrm{ml}$ for the diagnosis of probable/proven IFI. ${ }^{6}$ Many studies from Western countries have reported high specificity (>90\%) at the cutoff value of $80 \mathrm{pg} / \mathrm{mL} .{ }^{8,11,20}$ A metaanalysis study showed best diagnostic accuracy at the kit cutoff of $80 \mathrm{pg} / \mathrm{mL}(\mathrm{AOC}=0.885)$ with sensitivity of $78 \%$ and specificity of $81 \%{ }^{21}$ Compared with the Western countries, the environmental factors, fungal disease prevalence, and patient characteristics vary in the Indian population; it is important to understand the performance of these tests in Indian settings.

In our study, 17 patients on antifungal treatment were tested again after 3 to 4 weeks and 15 patients showed a decline in levels of BDG assay. A study from the United States monitored the prognostic response of the assay and found decreased levels are associated with successful treatment, while levels are increasing in case of treatment failure. ${ }^{22}$

Most of the critically ill patients in the hospital are already on antibiotics. A significant association was observed with dialysis and PTZ use. Other studies have demonstrated PTZ, amoxicillin-clavulanic acid, and dialysis as confounding factor and have shown reduced BDG levels on discontinuation of these factors. ${ }^{9,10,23,24}$ However, we could not observe the BDG levels after discontinuation of these factors by repeat test. This could be one of the reasons for higher cutoff in the study.

In the present study, $8.2 \%$ of the patients had a fatal outcome. A higher mortality rate $(25.7 \%)$ has been observed in a study conducted by Singh et al. ${ }^{6}$ In a French study, $18 \%$ mortality was seen in patients with IFI. ${ }^{25} \mathrm{~A}$ meta-analysis study showed $49.2 \%$ pooled mean mortality in candidemia patients. ${ }^{12}$ One of the reasons behind decreased mortality in our study might be the early start of empirical antifungal treatment and regulated use of antibiotics based on the hospital antibiotic policy.

\section{Conclusion}

Fungal biomarkers such as BDG are a ray of hope for rapid screening of fatal IFI in critically ill patients. As the assay has a high NPV, a negative test can be used to stop the empirical antifungal drugs. Keeping in mind the confounding factors and use of higher cutoffs can be useful to avoid false-positive results. Serial testing may be helpful in the prognosis of treatment. Compared with the Western countries, the environmental factors, including the fungal spore burden, fungal disease prevalence, and patient characteristics vary in the Indian population. Therefore, it is important to understand the performance of these tests in Indian settings by performing more clinical and epidemiological studies in these settings.

\section{Note}

The study has been approved by ethics committee of the institute.

\section{Conflict of Interest \\ None declared.}

\section{References}

1 Paramythiotou E, Frantzeskaki F, Flevari A, Armaganidis A, Dimopoulos $\mathrm{G}$. Invasive fungal infections in the ICU: how to approach, how to treat. Molecules 2014;19(01):1085-1119

2 Lamoth F, Akan H, Andes D, et al. Assessment of the role of 1,3- $\beta$ $\mathrm{d}$-Glucan testing for the diagnosis of invasive fungal infections in adults. Clin Infect Dis 2021;72(Suppl 2):S102-S108

3 Hou TY, Wang SH, Liang SX, Jiang WX, Luo DD, Huang DH. The screening performance of serum 1,3-beta-D-glucan in patients with invasive fungal diseases: a meta-analysis of prospective cohort studies. PLoS One 2015;10(07):e0131602

4 Xiaoling L, Tingyu T, Caibao H, Tian Z, Changqin C. Diagnostic efficacy of serum 1,3- $\beta$-D-glucan for invasive fungal infection: an update meta-analysis based on 37 case or cohort studies. Open Med (Wars) 2018;13:329-337

5 Pickering JW, Sant HW, Bowles CA, Roberts WL, Woods GL. Evaluation of a (1->3)-beta-D-glucan assay for diagnosis of invasive fungal infections. J Clin Microbiol 2005;43(12): 5957-5962

6 Singh S, Kaur H, Choudhary H, et al. Evaluation of biomarkers: galactomannan and 1,3-beta-D-glucan assay for the diagnosis of invasive fungal infections in immunocompromised patients from a tertiary care centre. Indian J Med Microbiol 2018;36(04): 557-563

7 Miyazaki T, Kohno S, Mitsutake K, et al. Plasma (1->3)-beta-Dglucan and fungal antigenemia in patients with candidemia, aspergillosis, and cryptococcosis. J Clin Microbiol 1995;33(12): 3115-3118

8 Tran T, Beal SG. Application of the 1,3- $\beta$-D-glucan (Fungitell) assay in the diagnosis of invasive fungal infections. Arch Pathol Lab Med 2016;140(02):181-185

9 Liss B, Cornely OA, Hoffmann D, Dimitriou V, Wisplinghoff H. 1,3$\beta$-D-Glucan contamination of common antimicrobials. J Antimicrob Chemother 2016;71(04):913-915

10 Kato A, Takita T, Furuhashi M, Takahashi T, Maruyama Y, Hishida A. Elevation of blood (1->3)-beta-D-glucan concentrations in hemodialysis patients. Nephron 2001;89(01):15-19

11 Alexander BD, Smith PB, Davis RD, Perfect JR, Reller LB. The $(1,3)$ beta-D-glucan test as an aid to early diagnosis of invasive fungal 
infections following lung transplantation. J Clin Microbiol 2010; 48(11):4083-4088

12 Zhang Z, Zhu R, Luan Z, Ma X. Risk of invasive candidiasis with prolonged duration of ICU stay: a systematic review and metaanalysis. BMJ Open 2020;10(07):e036452

13 Herbrecht R, Bories P, Moulin JC, Ledoux MP, Letscher-Bru V. Risk stratification for invasive aspergillosis in immunocompromised patients. Ann N Y Acad Sci 2012;1272:23-30

14 Bansal N, Gopalakrishnan R, Sethuraman N, et al. Experience with $\beta$-d-glucan assay in the management of critically ill patients with high risk of invasive candidiasis: an observational study. Indian J Crit Care Med 2018;22(05):364-368

15 Chandrasekar P. Diagnostic challenges and recent advances in the early management of invasive fungal infections. Eur J Haematol 2010;84(04):281-290

16 Cordonnier C, Botterel F, Ben Amor R, et al. Correlation between galactomannan antigen levels in serum and neutrophil counts in haematological patients with invasive aspergillosis. Clin Microbiol Infect 2009;15(01):81-86

17 Kaur S, Gupta V, Chhina DK, Singh A, Sharma D. Mycological and serological study of invasive Aspergillosis in a tertiary care hospital. J Microbiol Infect Dis 2018;8:8-12

18 Kritikos A, Poissy J, Croxatto A, Bochud PY, Pagani JL, Lamoth F. Impact of the beta-glucan test on management of intensive care unit patients at risk for invasive candidiasis. J Clin Microbiol 2020; 58(06):e01996-e19
19 Mikulska M, Furfaro E, Del Bono V, et al. Persistence of a positive (1,3)-beta-D-glucan test after clearance of candidemia in hematopoietic stem cell transplant recipients. Clin Vaccine Immunol 2011;18(03):518-519

20 Persat F, Ranque S, Derouin F, Michel-Nguyen A, Picot S, Sulahian A. Contribution of the (1->3)-beta-D-glucan assay for diagnosis of invasive fungal infections. J Clin Microbiol 2008;46(03): 1009-1013

21 He S, Hang JP, Zhang L, Wang F, Zhang DC, Gong FH. A systematic review and meta-analysis of diagnostic accuracy of serum $1,3-\beta$ D-glucan for invasive fungal infection: focus on cutoff levels. J Microbiol Immunol Infect 2015;48(04):351-361

22 Jaijakul S, Vazquez JA, Swanson RN, Ostrosky-Zeichner L. (1,3)- $\beta$ $D$-glucan as a prognostic marker of treatment response in invasive candidiasis. Clin Infect Dis 2012;55(04):521-526

23 Mennink-Kersten MA, Warris A, Verweij PE. 1,3-beta-D-glucan in patients receiving intravenous amoxicillin-clavulanic acid. $\mathrm{N}$ Engl J Med 2006;354(26):2834-2835

24 Leleu G, Aegerter P, Guidet BCollège des Utilisateurs de Base de Données en Réanimation. Systemic candidiasis in intensive care units: a multicenter, matched-cohort study. J Crit Care 2002;17 (03):168-175

25 Herbrecht R, Caillot D, Cordonnier C, et al. Indications and outcomes of antifungal therapy in French patients with haematological conditions or recipients of haematopoietic stem cell transplantation. J Antimicrob Chemother 2012;67(11):2731-2738 